\title{
Diffusion of a Janus nanoparticle in an explicit solvent: A molecular dynamics simulation study
}

\author{
Ali Kharazmi ${ }^{1}$ and Nikolai V. Priezjev ${ }^{2}$ \\ ${ }^{1}$ Department of Mechanical Engineering, \\ Michigan State University, East Lansing, Michigan 48824 and \\ ${ }^{2}$ Department of Mechanical and Materials Engineering, \\ Wright State University, Dayton, Ohio 45435
}

(Dated: November 16, 2018)

\begin{abstract}
Molecular dynamics simulations are carried out to study the translational and rotational diffusion of a single Janus particle immersed in a dense Lennard-Jones fluid. We consider a spherical particle with two hemispheres of different wettability. The analysis of the particle dynamics is based on the time-dependent orientation tensor, particle displacement, as well as the translational and angular velocity autocorrelation functions. It was found that both translational and rotational diffusion coefficients increase with decreasing surface energy at the nonwetting hemisphere, provided that the wettability of the other hemisphere remains unchanged. We also observed that in contrast to homogeneous particles, the nonwetting hemisphere of the Janus particle tends to rotate in the direction of the displacement vector during the rotational relaxation time.
\end{abstract}

PACS numbers: 68.08.-p, 66.20.-d, 83.10.Rs 


\section{INTRODUCTION}

Understanding the processes of self- and directed-assembly of nanoparticles is important for design of various nanostructured materials with advantageous mechanical and optical properties [1]. Recent progress in synthesis of Janus and asymmetric patchy particles, that can form a variety of predictable superstructures [2 [5], makes their production feasible at an industrial scale [6, 7]. The dynamics of assembly depends on the particle structure, distribution of wettability and charges, and boundary conditions at the particle surface in a solvent. The hydrodynamic boundary conditions are usually specified via the so-called slip length, which is defined as an extrapolated distance with respect to the interface where the velocity profile vanishes [8]. In general, it was shown that the slip length is a tensor quantity for flows over anisotropic textured surfaces [9-12]. Transport properties of chemically homogeneous nanoparticles were recently studied in cylindrical pores filled with a wetting fluid [13] and in an atomistic solvent confined between flat walls [14]. However, the diffusion process of individual Janus particles even in the absence of flow or confinement remains not fully understood.

At the continuum level, the hydrodynamic behavior of Janus particles was studied in the low Reynolds number regime [15-18]. It was found that when the slip length is smaller than the particle size, the translational velocity of the spherical Janus particle is coupled to the moments of the force density on its surface [15]. Moreover, it was shown that due to its asymmetrical boundary conditions, Janus particles can migrate parallel to the velocity gradient in shear flows [15]. In the limit of small slip lengths, the torque and force on spherical Janus particles with either discontinuous binary or continuously patterned surfaces were computed [16, 17]. More recently, torques and forces on pill-shaped Janus particles with stick and slip boundary conditions were analyzed using the boundary integral method [18]. In particular, it was demonstrated that depending on the aspect ratio and slip length, the force transverse to the direction of a uniform flow can change sign [18]. In the present work, the diffusive motion of a Janus particle in a quiescent fluid was studied using molecular dynamics (MD) simulations.

In recent years, Brownian motion of spherical particles with uniform wettability was extensively studied by MD simulations [19 26]. It was shown that a number of parameters, e.g., the mass and size ratios of a particle and a solvent molecule, interaction potentials, 
boundary conditions and the system size, can influence the diffusion process. Notably, at long times, the characteristic algebraic decay of the velocity autocorrelation function (VACF) due to hydrodynamic correlation between a particle and a fluid [27] depends on the particle diameter [22]; while at short times, the VACF exhibits pronounced oscillations at small values $(\lesssim 10)$ of the mass ratio between the particle and the fluid molecule [19, 24]. It was also found that when the ratio of the system size to the particle radius is larger than about 20, then the finite size effects due to particle images are not important [23, 25]. Furthermore, in the case of a smooth spherical particle, the Stokes-Einstein relation for the diffusion coefficient holds for the system with slip boundary conditions [19]. However, if the particle consists of a cluster of atoms and their interaction energy with fluid molecules is sufficiently large, then stick boundary conditions apply at the particle surface [23, 25].

In this paper, the results of molecular dynamics simulations are reported for the translational and rotational diffusion of individual Janus nanoparticles in a quiescent solvent. In our study, Janus particles are modeled as spheres with two sides of different wettability, and their behavior is contrasted with that of uniformly wetting and nonwetting particles. We find that both translational and rotational diffusion coefficients are larger for Janus particles with lower surface energy at the nonwetting hemisphere, provided that the wettability of the other hemisphere remains the same. It also will be shown that long-time algebraic decay of the velocity autocorrelation functions is recovered for uniform and anisotropic particles. Interestingly, the diffusive motion of Janus particles involves a subtle correlation between translational and angular velocities; i.e., a non-zero velocity component parallel to the particle symmetry plane causes a finite rotation to reduce drag.

The rest of the paper is structured as follows. The description of the molecular dynamics simulation model and parameter values is given in the next section. The analysis of the fluid density profiles, boundary conditions, particle displacement and rotation, as well as translational and angular velocity autocorrelation functions is presented in Sec.III. The conclusions are provided in the last section.

\section{MOLECULAR DYNAMICS SIMULATION MODEL}

The diffusive motion of a Janus particle in an explicit solvent was studied using classical molecular dynamics simulations [32]. In the explicit solvent model, the pairwise interaction 
between fluid monomers is specified via the truncated Lennard-Jones (LJ) potential

$$
V_{L J}(r)=4 \varepsilon\left[\left(\frac{\sigma}{r}\right)^{12}-\left(\frac{\sigma}{r}\right)^{6}\right]
$$

where $\varepsilon$ is the depth of the potential well and $\sigma$ is the separation distance at which the LJ potential is zero. The cutoff radius was fixed to $r_{c}=2.5 \sigma$ for all types of interactions. The system consists of 46531 fluid monomers and a Janus particle, which are confined into a cubic box of linear size $39.62 \sigma$. All simulations were performed at a constant volume with the fluid density $\rho=0.75 \sigma^{-3}$ and temperature $1.1 \varepsilon / k_{B}$, where $k_{B}$ is the Boltzmann constant. The fluid temperature was maintained by the Nosé-Hoover thermostat with the coupling parameter of 1.0. Periodic boundary conditions were applied in all three directions. The equations of motion were integrated numerically using the Verlet algorithm [30] with a time step $\triangle t_{M D}=0.005 \tau$, where $\tau=\sigma \sqrt{m / \varepsilon}$ is the time scale of the LJ potential.

The Janus particle was modeled as a convex polyhedron, the so-called Pentakis snub dodecahedron, which consists of 140 triangular faces, 210 edges, and 72 vertices [31]. Out of the total 210 edges, there are 60 short $(0.86 \sigma)$ and 150 long $(0.99 \sigma)$ edges. The radius of a sphere that contains all vertices is $2.08 \sigma$. In our model, $72 \mathrm{LJ}$ atoms were fixed relative to each other at the vertices of the polyhedron; and, during the simulations, the Janus particle was moving as a rigid body under forces from the surrounding fluid. The particle atoms do not interact with each other and their size is the same as the size of fluid monomers. The particle mass is $M=50.0 \mathrm{~m}$. The surface of the Janus particle was divided into two hemispheres of different wettability, as shown in Fig.1, The LJ interaction energy between fluid monomers and particle atoms at the wetting hemisphere was fixed to $\varepsilon_{\mathrm{pf}}=1.0 \varepsilon$, and at the nonwetting hemisphere $\varepsilon_{\mathrm{pf}}$ was varied in the range $0.1 \varepsilon \leqslant \varepsilon_{\mathrm{pf}} \leqslant 1.0 \varepsilon$. The two limiting cases of uniformly wetting and nonwetting particles correspond to the values of the interaction energy with the fluid molecules of $1.0 \varepsilon$ and $0.1 \varepsilon$, respectively.

The Janus particle and the surrounding fluid phase were first equilibrated for about $2 \times 10^{7} \mathrm{MD}$ steps (or $\left.10^{5} \tau\right)$ and then the data were accumulated during the next $6 \times 10^{8}$ MD steps (or $3 \times 10^{6} \tau$ ). During the production runs, the position and orientation of the Janus particle, the velocity of all particle atoms as well as the position of the center of mass of the solvent were saved every $20 \mathrm{MD}$ steps. To accumulate statistics, the simulations were repeated in forty independent systems for each value of $\varepsilon_{\mathrm{pf}}$. In each case, the velocities of fluid monomers were initialized using the Maxwell-Boltzmann probability distribution with 
zero total linear and angular momenta [32]. We also checked that after equilibration, the Maxwell-Boltzmann distribution for the translational and angular velocities of the Janus particle is recovered.

\section{RESULTS}

It has long been recognized that in the presence of a smooth substrate, the fluid monomers tend to form several distinct layers parallel to the substrate [33]. The amplitude of the fluid density profiles gradually decays away from the substrate to a uniform bulk density profile. Similarly, in the case of a spherical particle, the spatial distribution of fluid monomers around the particle consists of several concentric layers [38]. In our simulations, the radial distribution function for fluid monomers is shown in Fig.2 for uniformly wetting $\left(\varepsilon_{\mathrm{pf}}=1.0 \varepsilon\right)$ and uniformly nonwetting $\left(\varepsilon_{\mathrm{pf}}=0.1 \varepsilon\right)$ particles. In both cases, the fluid density profiles decay to a uniform profile within about $(3-4) \sigma$ from the particle surface. As expected, fluid layering is more pronounced for the uniformly wetting particle. In simulations with a Janus particle, a reduced value of the surface energy at the nonwetting hemisphere resulted in a lower amplitude of the local fluid density profile above the nonwetting side, while the density oscillations remain large above the wetting hemisphere where the interaction energy is fixed to $\varepsilon$ (see the inset in Fig.2).

The flow boundary conditions at the particle surface depend on the atom density and the LJ interaction energy between particle atoms and fluid monomers. In order to estimate the Navier slip length, we performed a set of separate simulations of fluid flow (without a particle) confined between flat crystalline walls with the same density as the Janus particle. In this setup, the fluid phase with density $\rho=0.75 \sigma^{-3}$ is in contact with (111) planes of the face-centered cubic (fcc) lattice. Similar to the previous studies [39, 40], the Poiseuille flow was induced by a constant force $f_{x}=0.005 \varepsilon / \sigma$ applied to each fluid monomer in the direction parallel to the walls. The slip lengths were computed from a parabolic fit of the averaged velocity profiles for different values of the wall-fluid interaction energy. The results are summarized in TableI. It is evident that at high interaction energies, the slip length is about the particle radius, while at low energies the slip length becomes larger than the particle diameter. Note also that for flows over curved surfaces the slip length should be modified by the local radius of curvature [34 37 ]. 
In a quiescent solvent, the Janus particle undergoes translational and rotational diffusion under random forces from the fluid monomers. We start the discussion of our results by considering the position vector of the particle center of mass. The mean square displacement (MSD) for uniformly (non)wetting and Janus particles is plotted in Fig. 3 for different surface energies. In all cases, the center of mass of the fluid phase was subtracted from the particle position. It can be clearly seen that after the ballistic regime, the MSD increases linearly with time (as indicated by the dashed line in Fig. 3). As expected, the slowest and fastest diffusion correspond to uniformly wetting and nonwetting particles, respectively. In between, the displacement increases with decreasing surface energy at the nonwetting hemisphere of Janus particles. Overall, this result is not surprising and can be expected for any spatial distribution of binary wettability on the particle surface. The translational diffusion coefficient was computed from the relation $r^{2}=6 D_{t} t$ at times $t \gtrsim 10^{3} \tau$ (shown in the upper inset of Fig. 30).

The rotational dynamics of nanoparticles can be probed by analyzing the decay of the autocorrelation function of the unit vectors $\mathbf{e}_{i}$ that are fixed relative to the particle. In what follows, we choose the vector $\mathbf{e}_{1}$ along the main axis of symmetry of the particle (see Fig.11). The autocorrelation function $\left\langle\mathbf{e}_{1}(0) \cdot \mathbf{e}_{1}(t)\right\rangle$ is plotted in Fig. 4 for the Janus and uniformly wetting and nonwetting particles. One can observe a similar trend as with the translational diffusion; namely, with increasing surface energy at the nonwetting hemisphere, the rotational relaxation slows down, and the two limiting cases of uniformly (non)wetting particles correspond to (fastest) slowest decay. A typical rotational relaxation time, $\tau_{r}$, was estimated from an fit to a simple exponential decay, i.e.,

$$
\left\langle\mathbf{e}_{1}(0) \cdot \mathbf{e}_{1}(t)\right\rangle=\exp \left(-t / \tau_{r}\right)
$$

The results are reported in the inset of Fig. 4. It can be observed that $\tau_{r}$ gradually increases with increasing surface energy $\varepsilon_{\text {pf }}$, which implies that the rotational diffusion coefficient is larger for Janus particles with lower surface energy at the nonwetting hemisphere.

In order to emphasize the difference between uniform and anisotropic particles, we next consider the translational and angular velocity autocorrelation functions (VACF) defined as $\langle\mathbf{v}(0) \cdot \mathbf{v}(t)\rangle$ and $\langle\boldsymbol{\omega}(0) \cdot \boldsymbol{\omega}(t)\rangle$, respectively. First, we plot the translational VACF in Fig. 5 for uniformly (non)wetting and Janus particles. Note that in all cases, the VACF is determined by the thermal velocity $\mathbf{v}^{2}=3 k_{B} T / M$ at short times, while the algebraic decay 
with the slope -1.5 is recovered at long times, which is consistent with the previous MD results [22, 25, 27]. In the intermediate regime, the VACF is smaller for Janus particles with higher interaction energy at the nonwetting hemisphere. Similarly, the angular VACF for Janus particles gradually varies between the two limiting cases of uniformly wetting and nonwetting particles (shown in Fig.6). Although the statistics at long times is limited, it appears that the angular VACFs exhibit a power-law decay with the slope -2.5, in agreement with earlier predictions [28, 29].

The autocorrelation functions discussed above do not necessarily reflect the broken symmetry of Janus particles; in principle, one can expect an enhanced diffusion with decreasing surface wettability regardless of its spatial distribution. In what follows, we explore a correlation between translational and rotational motion of Janus particles. It is intuitively clear that if a constant force is applied to a Janus particle in a viscous fluid then the nonwetting face will be oriented along the direction of motion in order to reduce drag. In analogy, when a Janus particle moves under random forces from the surrounding fluid, its nonwetting hemisphere tends to rotate in the direction of the displacement vector. This effect can be quantified by computing a scalar product of the displacement vector of the center of mass during the time interval $t$ and a vector difference between unit vectors $\mathbf{e}_{1}(t)$ and $\mathbf{e}_{1}(0)$, and then plotting it as a function of time (not shown). However, we found that it is difficult to obtain good statistics for this quantity and, more importantly, to provide a clear physical interpretation of the effect.

Alternatively, a more direct way to probe the correlation between translation and orientation of Janus particles is to compute a rotation angle along the direction of displacement. More specifically, we first choose a time interval $t$, compute the displacement vector of the center of mass $\triangle \mathbf{r}$, determine angles between $\left(\mathbf{e}_{1}(0), \triangle \mathbf{r}\right)$ and $\left(\mathbf{e}_{1}(t), \triangle \mathbf{r}\right)$, and then plot the difference between these angles as a function of time. In other words, we measure how the unit vector $\mathbf{e}_{1}$ rotates along but not around the displacement vector. The averaged rotation angle is plotted in Fig. [ 7 as a function of time for the Janus and uniformly wetting and nonwetting particles. It can be clearly observed that, within statistical uncertainty, particles with uniform distribution of wettability do not, on average, rotate along the displacement vector. In contrast, Janus particles undergo a finite rotation, which increases with decreasing surface energy at the nonwetting hemisphere. In each case, the rotation 
angle is maximum at times of about the rotational relaxation time (see inset in Fig.44). Note also that the maximum rotation angle in Fig.7 occurs at larger times for Janus particles with higher surface energy, which correlates with a slower relaxation of the autocorrelation function $\left\langle\mathbf{e}_{1}(0) \cdot \mathbf{e}_{1}(t)\right\rangle$ for Janus particles with higher $\varepsilon_{\text {pf }}$ reported in Fig. 4. As expected, at longer times the correlation gradually decays, although it remains nonzero even at times of about $10^{4} \tau$.

To further quantify the effect of rotation along the displacement, we also measured a correlation between the translational and angular velocities. The corresponding correlation functions were defined as a product $\left\langle v_{i}(0) \cdot \omega_{j}(t)\right\rangle$, where the velocity components with indices $i, j=1,2,3$ were computed along the unit vectors $\mathbf{e}_{i}$ (see Fig.1). As a representative case, we consider a Janus particle with the largest difference in wettability, $(1.0 \varepsilon, 0.1 \varepsilon)$, when the correlation effect is most pronounced. The averaged correlation functions are presented in Fig. 8 for selected pairs of indices. It can be seen that the only non-zero correlation functions are $\left\langle v_{2}(0) \cdot \omega_{3}(t)\right\rangle$ and $\left\langle v_{3}(0) \cdot \omega_{2}(t)\right\rangle$. Physically, it means that if a Janus particle has a translational velocity component in a direction parallel to the symmetry plane then it will tend to rotate its nonwetting hemisphere towards the displacement. Finally, we note that for uniformly wetting/nonwetting particles there is no preferred rotation with respect to translation, and, therefore, the cross correlation functions are zero at all times (not shown).

\section{CONCLUSIONS}

In summary, we investigated the translational and rotational diffusion of individual Janus nanoparticles in a dense fluid using molecular dynamics simulations. In our model, the Janus particle consists of atoms arranged on a sphere, which is divided in two hemispheres of different wettability. The interaction energy between particle atoms and fluid molecules is fixed at the wetting hemisphere, while the wettability of the other side is a variable

parameter. The diffusion process of such particles is compared against two limiting cases of uniformly wetting and nonwetting particles.

We found that the diffusion coefficient of Janus particles is bounded between the two limiting cases and it increases with decreasing surface energy at the nonwetting hemisphere. The analysis of the translational and angular velocity autocorrelation functions showed that the exponents of the long-time power-law decay are the same for Janus and uniform particles. 
Perhaps most interestingly, we observed a correlation between translation and rotation of Janus particles; in other words, when a particle spontaneously moves in a particular direction it tends to reduce drag by rotating its nonwetting side towards the displacement vector. This effect is absent for homogeneous particles.

In the future, it will be instructive to extend these results to a system of many particles and study in detail the dynamics of self-assembly of Janus particles into complex structures in the bulk or at liquid-liquid interfaces.

\section{Acknowledgments}

Financial support from the National Science Foundation (CBET-1033662) is gratefully acknowledged. Computational work in support of this research was performed at Michigan State University's High Performance Computing Facility and the Ohio Supercomputer Center. The molecular dynamics simulations were conducted using the LAMMPS numerical code [32].

[1] Y. Min, M. Akbulut, K. Kristiansen, Y. Golan, and J. Israelachvili, Nature Materials 7, 527 (2008).

[2] Z. Zhang and S. C. Glotzer, Nano Letters 4, 1407 (2004).

[3] L. Hong, A. Cacciuto, E. Luijten, and S. Granick, Langmuir 24, 621 (2008).

[4] G. Rosenthal, K. E. Gubbins, and S. H. L. Klapp, J. Chem. Phys. 136, 174901 (2012).

[5] Z.-W. Li, Z.-Y. Lu, Z.-Y. Sun, and L.-J. An, Soft Matter 8, 6693 (2012).

[6] G. Loget and A. Kuhn, J. Mater. Chem. 22, 15457 (2012).

[7] S. Jiang, Q. Chen, M. Tripathy, E. Luijten, K. S. Schweizer, and S. Granick, Adv. Mater. 22, 1060 (2010).

[8] P. G. de Gennes, Rev. Mod. Phys. 57, 827 (1985).

[9] M. Z. Bazant, and O. I. Vinogradova, J. Fluid Mech. 613, 125 (2008).

[10] N. V. Priezjev, A. A. Darhuber, and S. M. Troian, Phys. Rev. E 71, 041608 (2005).

[11] O. I. Vinogradova and A. V. Belyaev, J. Phys.: Condens. Matter 23, 184104 (2011).

[12] N. V. Priezjev, J. Chem. Phys. 135, 204704 (2011). 
[13] G. Drazer, B. Khusid, J. Koplik, and A. Acrivos, Phys. Fluids 17, 017102 (2005).

[14] S. C. Kohale and R. Khare, J. Chem. Phys. 132, 234706 (2010).

[15] A. S. Khair and J. W. Swan, J. Fluid Mech. 606, 115 (2008).

[16] G. R. Willmott, Phys. Rev. E 77, 055302R (2008).

[17] G. R. Willmott, Phys. Rev. E 79, 066309 (2009).

[18] Q. Sun, E. Klaseboer, B. C. Khoo, and D. Y. C. Chan, Phys. Rev. E 87, 043009 (2013).

[19] F. Ould-Kaddour and D. Levesque, Phys. Rev. E 63, 011205 (2000).

[20] J. R. Schmidt and J. L. Skinner, J. Chem. Phys. 119, 8062 (2003).

[21] S. H. Lee and R. Kapral, J. Chem. Phys. 121, 11163 (2004).

[22] F. Ould-Kaddour and D. Levesque, J. Chem. Phys. 127, 154514 (2007).

[23] Z. Li, Phys. Rev. E 80, 061204 (2009).

[24] H. K. Shin, C. Kim, P. Talkner, and E. K. Lee, Chem. Phys. 375, 316 (2010).

[25] D. Chakraborty, Eur. Phys. J. B 83, 375 (2011).

[26] V. Y. Rudyak, S. L. Krasnolutskii, and D. A. Ivanov, Microfluid. Nanofluid. 11, 501 (2011).

[27] B. Alder and T. Wainwright, Phys. Rev. A 1, 18 (1970).

[28] N. K. Ailawadi and B. J. Berne, J. Chem. Phys. 54, 3569 (1971).

[29] G. Subramanian, D. G. Levitt, and H. T. Davis, J. Stat. Phys. 15, 1 (1975).

[30] M. P. Allen and D. J. Tildesley, Computer Simulation of Liquids (Clarendon, Oxford, 1987).

[31] J. H. Conway, H. Burgiel, C. Goodman-Strauss, The Symmetries of Things (A K Peters/CRC Press, 2008).

[32] S. J. Plimpton, J. Comp. Phys. 117, 1 (1995); see also URL http://lammps.sandia.gov.

[33] J. N. Israelachvili, Intermolecular and surface forces, second edition, (Academic Press, San Diego, 1992).

[34] D. Einzel, P. Panzer, and M. Liu, Phys. Rev. Lett. 64, 2269 (1990).

[35] A. Niavarani and N. V. Priezjev, Phys. Rev. E 81, 011606 (2010).

[36] N. V. Priezjev and S. M. Troian, J. Fluid Mech. 554, 25 (2006).

[37] W. Chen, R. Zhang, and J. Koplik, Phys. Rev. E 89, 023005 (2014).

[38] J. R. Schmidt and J. L. Skinner, J. Phys. Chem. B 108, 6767 (2004).

[39] N. V. Priezjev, Phys. Rev. E 75, 051605 (2007).

[40] N. V. Priezjev, J. Chem. Phys. 127, 144708 (2007). 
Figures 


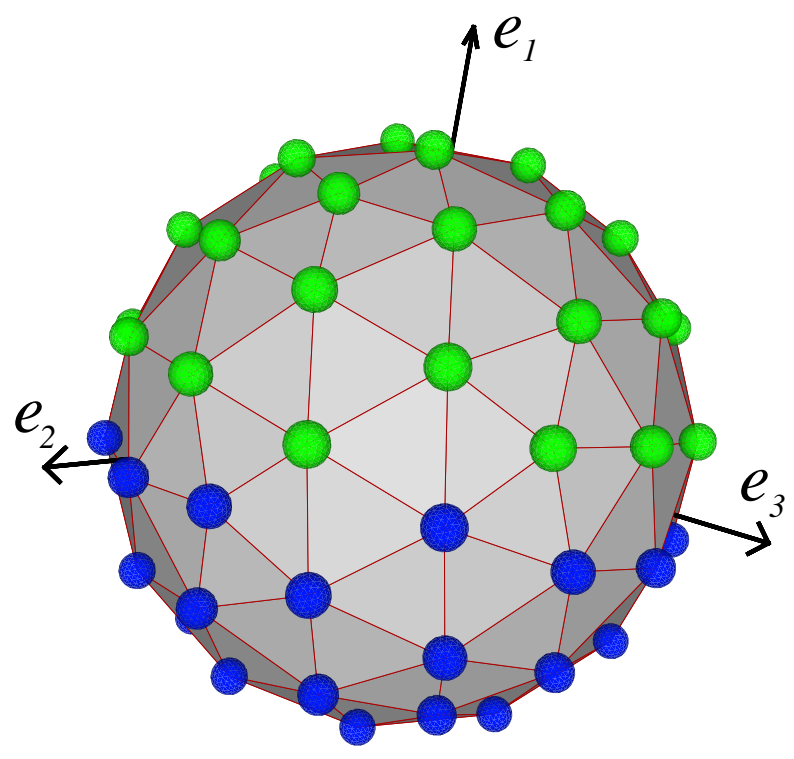

FIG. 1: (Color online) A snapshot of the Janus particle with wetting (blue atoms) and nonwetting (green atoms) hemispheres. The particle consists of 72 atoms rigidly fixed on vertices of a convex polyhedron (see text for details). Note that particle atoms are not drawn to scale. The unit vectors $\mathbf{e}_{1}, \mathbf{e}_{2}$ and $\mathbf{e}_{3}$ form an orthonormal basis in a reference frame of the particle. 


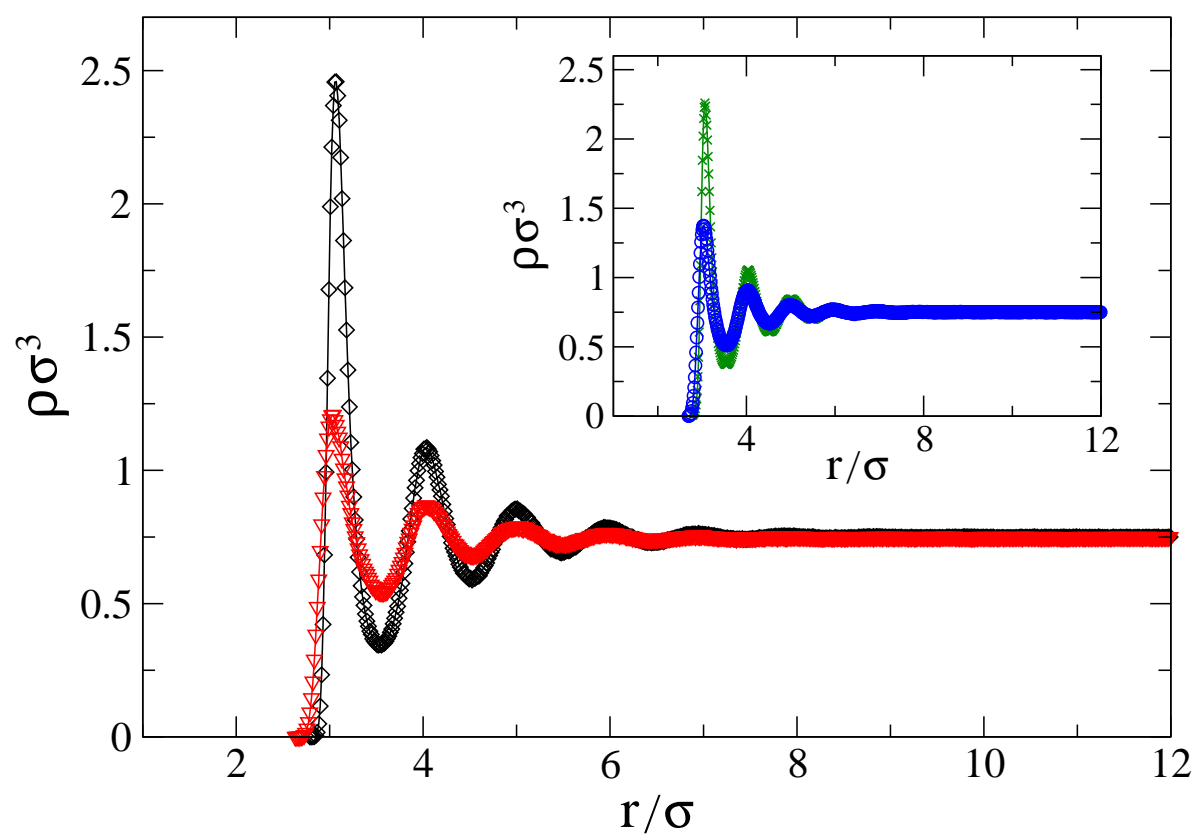

FIG. 2: (Color online) Averaged fluid density profiles as a function of the distance from the particle center for uniformly wetting $(\diamond)$ and uniformly nonwetting $(\nabla)$ particles. The LJ interaction energy between particle atoms and fluid monomers is $\varepsilon_{\mathrm{pf}}=1.0 \varepsilon(\diamond)$ and $\varepsilon_{\mathrm{pf}}=0.1 \varepsilon(\nabla)$. The inset shows fluid density profiles near the wetting hemisphere $\varepsilon_{\mathrm{pf}}=1.0 \varepsilon(\times)$ and nonwetting hemisphere $\varepsilon_{\mathrm{pf}}=0.1 \varepsilon(\circ)$ of the Janus particle. 


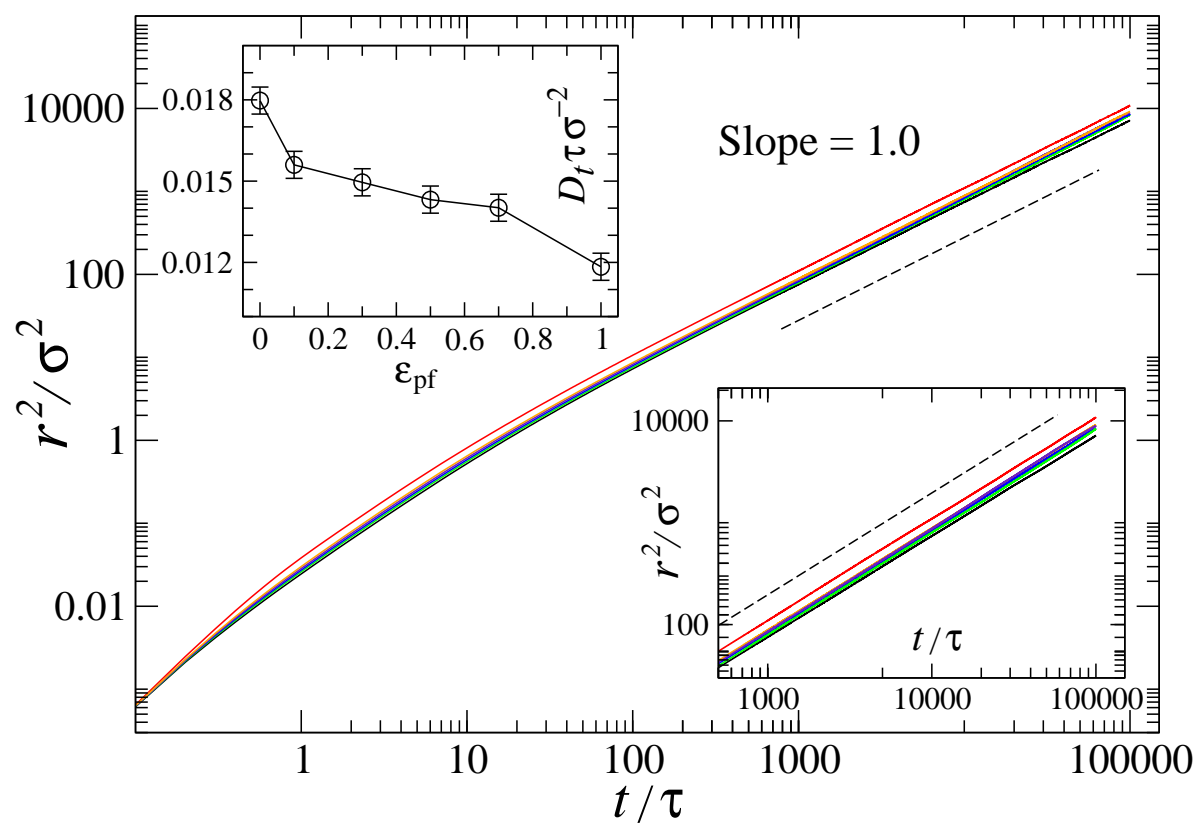

FIG. 3: (Color online) The mean square displacement of particles as a function of time for the surface energies at both hemispheres from top to bottom $(0.1 \varepsilon, 0.1 \varepsilon),(1.0 \varepsilon, 0.1 \varepsilon),(1.0 \varepsilon, 0.3 \varepsilon)$, $(1.0 \varepsilon, 0.5 \varepsilon),(1.0 \varepsilon, 0.7 \varepsilon)$, and $(1.0 \varepsilon, 1.0 \varepsilon)$. The dashed line with unit slope is plotted for reference. The lower inset shows an enlarged view of the same data at large times. The upper inset shows the diffusion coefficient as a function of $\varepsilon_{\mathrm{pf}}$, while the other hemisphere is wetting, i.e., $\varepsilon_{\mathrm{pf}}=1.0 \varepsilon$. The case $\varepsilon_{\mathrm{pf}}=0$ corresponds to a uniformly nonwetting particle $(0.1 \varepsilon, 0.1 \varepsilon)$. 


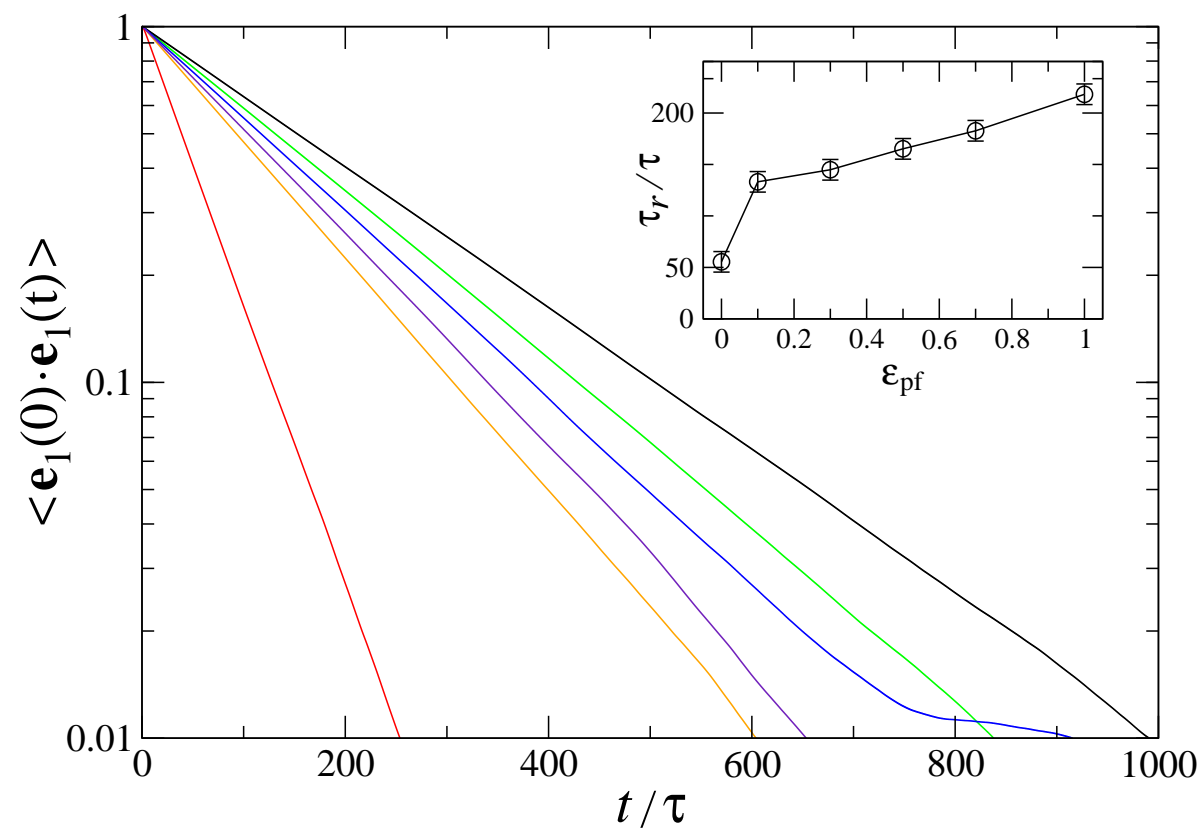

FIG. 4: (Color online) The autocorrelation function $\left\langle\mathbf{e}_{1}(0) \cdot \mathbf{e}_{1}(t)\right\rangle$ for the surface energies at both hemispheres $(0.1 \varepsilon, 0.1 \varepsilon),(1.0 \varepsilon, 0.1 \varepsilon),(1.0 \varepsilon, 0.3 \varepsilon),(1.0 \varepsilon, 0.5 \varepsilon),(1.0 \varepsilon, 0.7 \varepsilon)$, and $(1.0 \varepsilon, 1.0 \varepsilon)$ from left to right. The inset shows the rotational relaxation time $\tau_{r}$ (in units of $\tau$ ) as a function of the surface energy at the nonwetting hemisphere. 


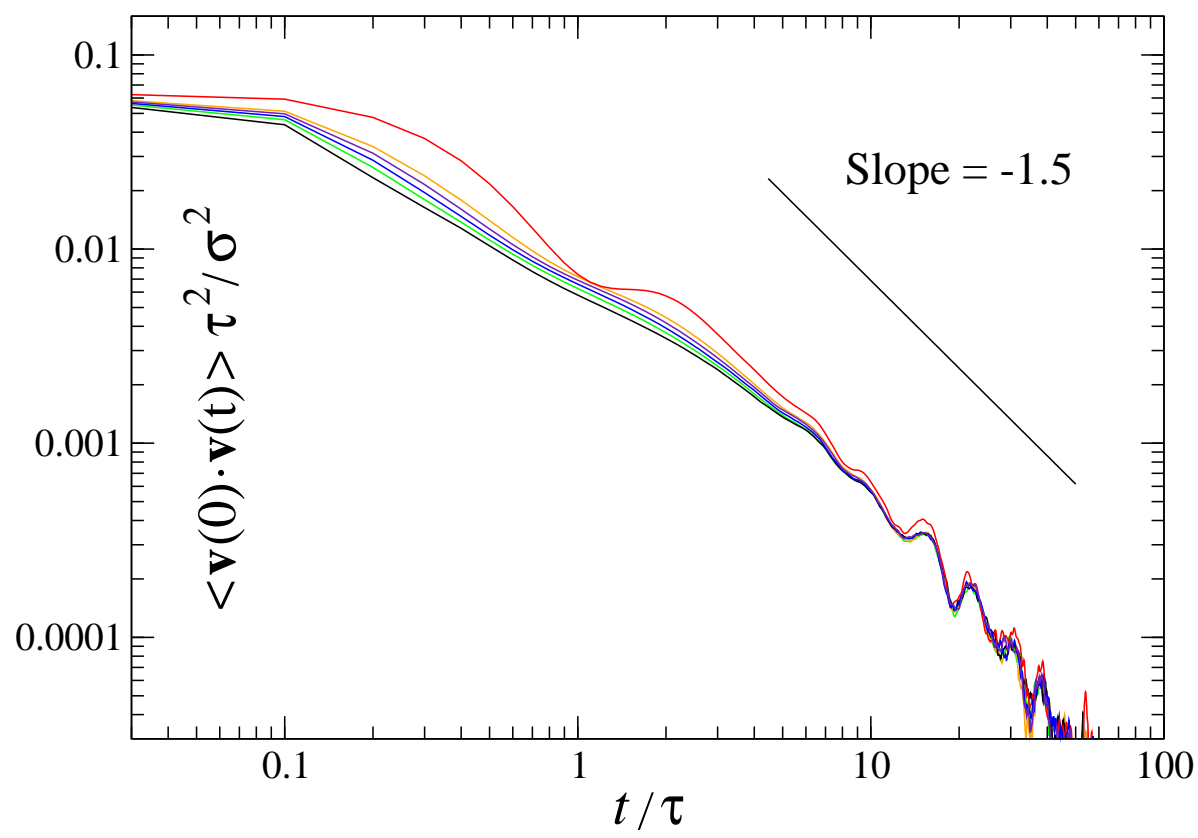

FIG. 5: (Color online) The velocity autocorrelation function $\langle\mathbf{v}(0) \cdot \mathbf{v}(t)\rangle$ for the interaction energies at both hemispheres $(0.1 \varepsilon, 0.1 \varepsilon),(1.0 \varepsilon, 0.1 \varepsilon),(1.0 \varepsilon, 0.3 \varepsilon),(1.0 \varepsilon, 0.5 \varepsilon),(1.0 \varepsilon, 0.7 \varepsilon)$, and $(1.0 \varepsilon$, $1.0 \varepsilon$ ) from top to bottom. The straight line with a slope -1.5 is shown as a reference. 


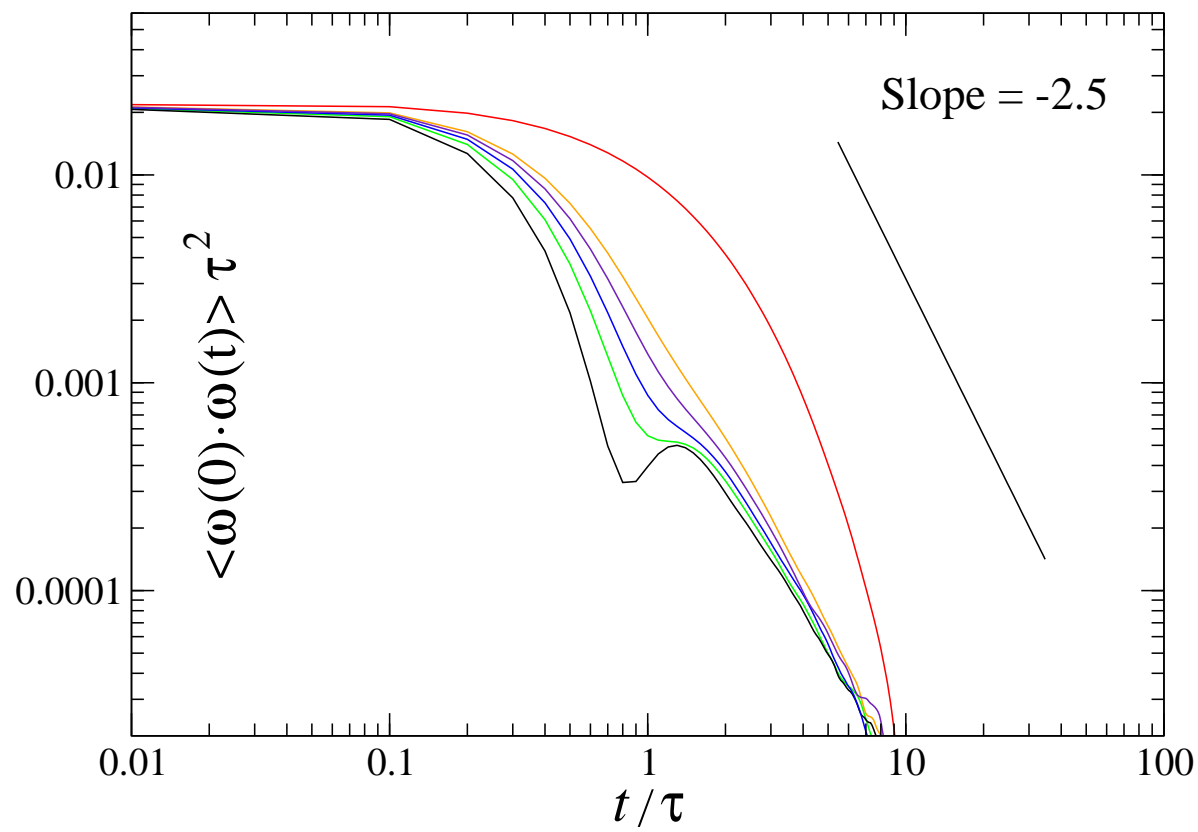

FIG. 6: (Color online) The angular velocity autocorrelation function $\langle\boldsymbol{\omega}(0) \cdot \boldsymbol{\omega}(t)\rangle$ for the LJ interaction energies at both hemispheres $(0.1 \varepsilon, 0.1 \varepsilon),(1.0 \varepsilon, 0.1 \varepsilon),(1.0 \varepsilon, 0.3 \varepsilon),(1.0 \varepsilon, 0.5 \varepsilon)$, $(1.0 \varepsilon, 0.7 \varepsilon)$, and $(1.0 \varepsilon, 1.0 \varepsilon)$ from top to bottom. The black line with a slope -2.5 is plotted for reference. 


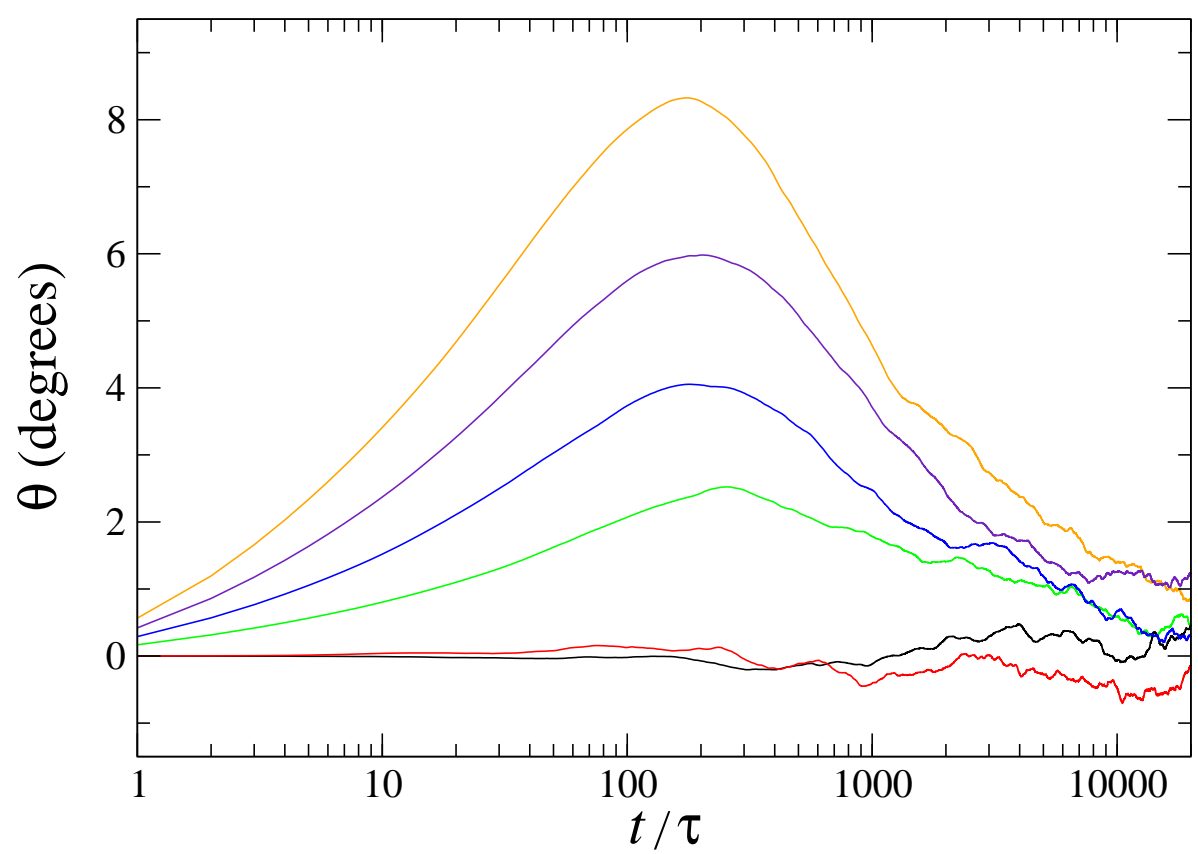

FIG. 7: (Color online) The rotation angle along the displacement vector of the center of mass for the surface energies at both hemispheres $(1.0 \varepsilon, 0.1 \varepsilon),(1.0 \varepsilon, 0.3 \varepsilon),(1.0 \varepsilon, 0.5 \varepsilon),(1.0 \varepsilon, 0.7 \varepsilon)$ from top to bottom. The data for uniformly wetting $(1.0 \varepsilon, 1.0 \varepsilon)$ and nonwetting $(0.1 \varepsilon, 0.1 \varepsilon)$ particles are indicated by the black and red curves, respectively. 


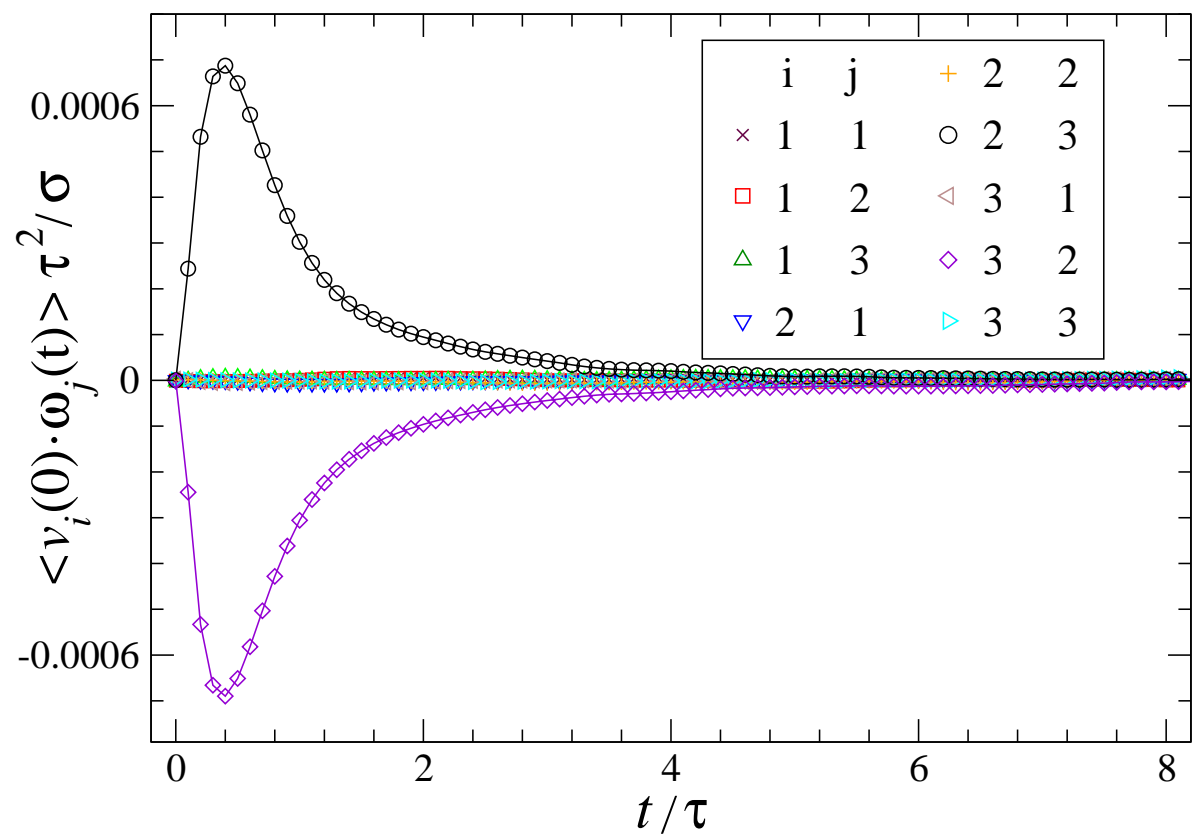

FIG. 8: (Color online) The translational times angular velocity correlation functions $\left\langle v_{i}(0) \cdot \omega_{j}(t)\right\rangle$ for the indicated pairs of indices. The velocity components are computed along the unit vectors $\mathbf{e}_{i}$ (see Fig.11). The data are reported for a Janus particle with the LJ interaction energies at both hemispheres $(1.0 \varepsilon, 0.1 \varepsilon)$. 
Tables 
TABLE I: The slip length $L_{s} / \sigma$ computed in a Poiseuille flow confined by flat crystalline walls with density $1.63 \sigma^{-3}$ for the tabulated wall-fluid interaction energies. The error bars for the slip length are about $0.5 \sigma$.

\begin{tabular}{lccccc}
\hline \hline$\varepsilon_{\mathrm{pf}} / \varepsilon$ & 0.1 & 0.3 & 0.5 & 0.7 & 1.0 \\
\hline$L_{s} / \sigma$ & 9.0 & 6.4 & 4.6 & 3.4 & 2.0 \\
\hline \hline
\end{tabular}

